\title{
Mundos Virtuais na prática docente: uma visão sobre os desafios e benefícios
}

\author{
Felipe Becker Nunes ${ }^{1}$, Fabrício Herpich ${ }^{1}$, Leo Natan Paschoal ${ }^{2}$, \\ Liane Margarida Rockenbach Tarouco ${ }^{1}$, José Valdeni de Lima ${ }^{1}$ \\ ${ }^{1}$ Centro Interdisciplinar de Novas Tecnologias na Educação - \\ Universidade Federal do Rio Grande do Sul \\ Caixa Postal 15.064 - 91.501-970 - Porto Alegre - RS - Brazil \\ ${ }^{2}$ Instituto de Ciências Matemáticas e de Computação - Universidade de São Paulo \\ Caixa Postal 668 - 13.566-970 - São Carlos - SP - Brasil \\ \{nunesfb, fabricio.herpich, leonatanpaschoal\}@gmail.com \\ liane@penta.ufrgs.br, valdeni@inf.ufrgs.br
}

\begin{abstract}
Virtual worlds can be considered highly interactive environments with a high degree of reality, being possible to use it in several areas of teaching. In this context, the present article sought to present an overview of the main challenges and benefits currently present in the use of this type of environment in teaching practice. A case study was applied along with a theoretical survey about the topic to address such issues. The results showed some of the main difficulties faced by teachers in using this environment, as well as highlighting the benefits that can be gained after overcoming such barriers.
\end{abstract}

Resumo. Os mundos virtuais podem ser considerados ambientes altamente interativos com um grau de realidade elevado, sendo possível utilizá-lo em diversas áreas de conhecimento. Neste contexto, o presente artigo busca apresentar uma visão geral dos principais desafios e benefícios presentes atualmente no uso deste tipo de ambiente na prática docente. Um estudo de caso foi realizado juntamente com um levantamento teórico sobre o tópico para abordar tais questões elencadas. Os resultados mostraram algumas das principais dificuldades enfrentadas pelos docentes ao utilizar este ambiente, assim como os benefícios que podem ser adquiridos após superar tais barreiras.

\section{Introdução}

No cenário tradicional de aprendizagem, cada indivíduo que compõe um grupo de alunos está sujeito às políticas e métodos de aprendizagem propostos pela instituição de ensino e/ou docentes. Atualmente, os estudantes pertencem a uma sociedade digital, pois nasceram no meio social tecnológico e estão acostumados com a linguagem hipermídiatica dos jogos digitais, redes sociais e ambientes virtuais.

Neste contexto, professores de diferentes níveis de ensino têm buscado alternativas para que possam usufruir de novas soluções tecnológicas existentes (e.g. jogos digitais, recursos de realidade aumentada, ambientes de aprendizagem), ao mesmo tempo que objetivam proporcionar ao aluno experiências inovadoras que unem a prática docente com a tecnologia. [Twining et al. 2013] abordam esta situação explicando que os professores 
VI Congresso Brasileiro de Informática na Educação (CBIE 2017)

Anais do XXIII Workshop de Informática na Escola (WIE 2017)

em formação têm um papel fundamental a desempenhar na introdução, desenvolvimento e apoio aos processos de ensino e aprendizagem inovadores, como parte da integração da tecnologia para atender às necessidades dos alunos do século 21.

Dentre as soluções adotadas, estão os mundos virtuais, que podem ser vistos como uma metáfora computacional do mundo que conhecemos, com pessoas, lugares e objetos [Zanotto et al. 2009]. Outra definição complementar é que são ambientes gráficos simulados por computador, no qual os seres humanos convivem com outros usuários através de seus avatares [Griol et al. 2014].

Nesta direção, conforme explicitado por [Johnson et al. 2009], os mundos virtuais podem oferecer um novo tipo de abordagem, na qual os usuários passam de um estado considerado passivo e observatório para se tornarem sujeitos mais ativos dentro do mundo virtual. A representação de objetos tridimensionais e a atribuição de movimentos para melhor exemplificar como ocorrem determinados fenômenos dentro do mundo virtual englobando áreas como física, química e ciências, tem um potencial instigador para captar a atenção dos alunos durante uma atividade educacional.

Neste tipo de ambiente, os laboratórios virtuais são uma alternativa aos laboratórios escolares na vida real, em que algumas experiências são inviáveis de serem realizadas devido a dimensão macro ou micro, riscos inerentes e recursos de infraestrutura. [Kozlovsky and Kravtsov 2011] explicam que os laboratórios virtuais podem ser considerados como um ambiente virtual em que a possibilidade de pesquisa de comportamentos de modelos de objetos, seus conjuntos e derivados, estabelecida com a participação certa de detalhamento em relação aos objetos reais.

Professores que têm implementado novas abordagens pedagógicas com o uso da tecnologia, são muitas vezes extremamente conscientes dos inúmeros problemas que surgem, quando se tenta fazer modificações em um ambiente tradicional de ensino [Jacka 2015]. Nessa perspectiva, a criatividade do professor também se torna indispensável para o planejamento e a construção dos laboratórios de ensino nos mundos virtuais, sendo necessária o uso de sua expertise para que o uso de simulações e recursos multimídias estejam adequados e operando de forma correta.

É nesse contexto que o presente artigo se insere, tendo o objetivo de descrever alguns dos principais desafios e benefícios envolvendo a prática docente na implementação dos mundos virtuais dentro ou fora da sala de aula. A motivação para o desenvolvimento deste trabalho está centralizado na constatação de pesquisas que sobreleva a importância de esclarecer os principais desafios e benefícios que os docentes experienciam ao utilizar mundos virtuais, além da expertise dos autores, que têm centralizado pesquisas nos últimos 6 anos no uso dos mundos virtuais.

Será apresentado um aporte teórico no decorrer deste artigo e a descrição de um estudo de caso envolvendo professores em formação no uso de um laboratório virtual voltado para o ensino de ciências ensinadas no ensino fundamental no contexto da educação básica ( $6^{\circ}$ ano até o $9^{\circ}$ ano). Com isso, serão abordados e analisados aspectos referentes desde o processo de construção e instalação do ambiente, assim como a capacitação dos docentes e a consequente implantação em sala de aula juntamente aos alunos envolvendo práticas e estratégias didáticas. 
VI Congresso Brasileiro de Informática na Educação (CBIE 2017)

Anais do XXIII Workshop de Informática na Escola (WIE 2017)

\section{Trabalhos Relacionados}

Pesquisas envolvendo a prática docente, de forma direta ou indireta, têm sido desenvolvidas buscando apresentar propostas e resultados obtidos envolvendo a intervenção docente no uso dos mundos virtuais. No trabalho de [Avila et al. 2014], uma apresentação introdutória acerca de algumas iniciativas envolvendo a capacitação docente é apresentado, cujo resultados serviram para propor uma nova estratégia de aprendizado dos docentes no que concerne à construção dos mundos virtuais.

Seguindo uma linha de pensamento similar ao artigo descrito anteriormente, o estudo de [Gregory et al. 2015] apresenta uma pesquisa de opinião envolvendo alunos e professores, sobre as principais barreiras e facilitadores presentes no uso dos mundos virtuais. A pesquisa apresenta dados quantitativos e qualitativos à discussão dos resultados obtidos, sendo que os autores concluíram que apesar das dificuldades inerentes à alunos e professores, muitas vezes pela falta de capacitação e erro do professor ao aplicar em sala de aula, os mundos virtuais têm um espaço instigador a ser explorado no futuro.

No trabalho de [Jacka 2015], o objetivo foi efetuar uma pesquisa que buscasse apresentar modelos de ensino e capacitação envolvendo o uso de mundos virtuais com professores em formação. Uma análise qualitativa com o uso de entrevista, questionários e observações foi utilizada, em que os resultados possibilitaram a criação de um modelo para os professores utilizarem como suporte e guia na implementação dos mundos virtuais na sua prática docente.

Baseado nas pesquisas apresentadas e demais trabalhos analisados na construção deste artigo, assim como no conhecimento empírico dos autores, se tem como principal diferencial a análise descritiva dos desafios docentes envolvendo o processo de criação do projeto até sua parte final que envolve a aplicação em sala de aula.

\section{Prática Docente e Mundos Virtuais}

Professores em formação e demais docentes com mais anos de experiência têm tido o desafio de se atualizarem e aceitarem a oscilação de mudanças em sala de aula no que concerne ao uso da Tecnologia na Educação, em que estes primeiros têm o benefício de serem considerados nativos digitais, ou seja, estarem vivendo em um meio altamente tecnológico e informatizado [Prensky 2001]. Em consonância com isto está o pensamento de [Fullan 2013], que entende que nas escolas tem ocorrido um efeito push-pull, em que tanto estudantes quanto professores estão sendo empurrados para longe da educação formal e puxados para a tecnologia fora da sala de aula.

É neste contexto que programas de capacitação e formação continuada em tecnologias na educação têm um papel fundamental, pois com a aplicação destes cursos em conjunto com a pró-atividade dos próprios professores, se torna possível criar oportunidades para eles construírem uma base consistente adequada a aplicação de novas soluções em sala de aula ou em atividades fora da sala de aula. No contexto desta pesquisa, a aplicação deste tipo de capacitação e atitude do professor é essencial quando diz respeito à utilização dos mundos virtuais na educação, visto que este envolve independente da área do professor, um maior conhecimento computacional.

[Jacka 2015] entende que ainda há algum senso acerca dos mundos virtuais de que estes estão em um processo de construção e muito trabalho ainda precisa ser realizado 
VI Congresso Brasileiro de Informática na Educação (CBIE 2017)

Anais do XXIII Workshop de Informática na Escola (WIE 2017)

para os professores em formação, estudantes e gestores adotarem plenamente os mundos virtuais como um espaço de aprendizagem. Conforme descrito anteriormente, se torna uma das principais dificuldades para o professor, principalmente se o uso do ambiente envolver a implementação e criação do mundo virtual, que ele adquira e saiba operar este tipo de recurso tecnológico.

Conforme descrito por [Nunes et al. 2016a], em uma revisão sistemática sobre mundos virtuais aplicados na educação, um número menor de ambientes utiliza recursos multimídias e simulações com o uso de scripts de programação, visto que a complexidade se torna maior e dificulta a sua utilização. Apesar da curva de aprendizagem não ser considerada elevada e custosa, se torna essencial para os docentes contornar está dificuldade integrando pessoas capacitadas que possam fornecer o suporte tecnológico necessário para que o ambiente esteja em pleno funcionamento.

Um ponto importante relacionado a isto é a infraestrutura tecnológica que se faz necessária para o funcionamento do mundo virtual, visto que o uso de servidores com uma conexão de Internet adequada é exigido para o correto funcionamento do ambiente. [Fernandes et al. 2007] destacam algumas preocupações envolvendo o tráfego de dados, download e streaming durante a interação dos usuários nos mundos virtuais. Por exemplo, os autores citam que o uso de voz, música, vídeo e dados em um único mundo virtual com uma quantidade significativa de usuários conectados de diferentes locais, pode impactar negativamente na qualidade dos serviços oferecidos. No lado do cliente, caso o usuário não detenha de um computador com adequados recursos para o pleno funcionamento do mundo virtual, isto pode agravar sua interação, gerando frustração e falta de motivação, o que pode comprometer seu retorno ao ambiente.

Tais pontos descritos são importantes para o professor considerar juntamente com pessoas capacitadas, no momento de utilizar os mundos virtuais em sala de aula ou em atividades a distância, devendo realizar um planejamento adequado da infraestrutura e do próprio ambiente. Desta forma, os usuários poderão usufruir das totais características presentes nos mundos virtuais, dentre as quais estão, suas características imersivas, sua possibilidade de customização dos diferentes espaços, personagens e contextualização de situações.

Paralelo ao ponto de vista estrutural e tecnológico, também existe outro lado que opera conjuntamente, sendo ele o planejamento e a definição das atividades didáticas propostas pelo professor no mundo virtual. É essencial que uma metodologia bem definida baseada em uma abordagem ou teoria educacional seja estabelecida, com objetivos bem claros e que a forma de aplicação esteja em harmonia com as características do mundo virtual, buscando usufruir adequadamente do potencial oferecido neste ambiente. [Nunes et al. 2016b] ressaltam que a autonomia fornecida com uma abordagem educacional bem definida pode auxiliar os estudantes no desenvolvimento de suas ações próprias e investigações acerca de novos conhecimentos calcados nos recursos presentes nestes ambientes tridimensionais (3D).

Exemplo disto pode ser visto no levantamento sistemático feito em [Nunes et al. 2016a], no qual são descritas diversas formas com que foi aplicada uma teoria educacional para realização de atividades didáticas nos mundos virtuais em diferentes áreas de ensino. [Christensen et al. 2013] ressaltam que o foco geralmente está 
VI Congresso Brasileiro de Informática na Educação (CBIE 2017)

Anais do XXIII Workshop de Informática na Escola (WIE 2017)

presente neste lado educacional a ser definido pelo professor, porém recordam que os recursos computacionais também precisam ser cuidadosamente estabelecidos e organizados para evitar possíveis problemas técnicos.

\section{Procedimentos Metodológicos}

A metodologia empregada nesta pesquisa pode ser dividida em diferentes etapas de aplicação, como são detalhadas a seguir. A primeira etapa consistiu em um levantamento teórico acerca dos Mundos Virtuais e sua aplicação na prática docente, assim como foi realizada uma análise de alguns trabalhos relacionados e os direcionamentos das pesquisas descritas.

É importante ressaltar que este artigo não tem como foco principal apresentar a estrutura tecnológica utilizada e nem a descrição detalhada do mundo virtual criado. Portanto, será apresentado de forma clara e breve as informações necessárias para o leitor situar-se, visando focar predominantemente nos desafios e benefícios envolvendo a prática docente na implementação dos mundos virtuais nesse experimento realizado.

A infraestrutura tecnológica utilizada para aporte desta pesquisa foi um servidor Web com endereço IP dedicado, em que o OpenSim (versão 0.8.1) foi selecionado por ser open source e com uma vasta documentação no meio acadêmico; para a visualização do mundo virtual foi selecionado o viewer Singularity, que possui recursos apropriados para atender às necessidades identificadas na construção do mundo virtual. O Wamp Server foi utilizado para gerenciar o acesso externo, o banco de dados do ambiente na plataforma MySQL e a linguagem Hypertext Preprocessor para programação dos scripts necessários.

No mundo virtual OpenSim, uma região foi estabelecida para hospedar o laboratório virtual a ser utilizado pelos professores. O laboratório virtual criado pertence a um projeto voltado para o ensino de ciências para alunos de séries fundamentais, sendo seu desenvolvimento efetuado juntamente com um professor que atua nesta área, ministrando a disciplina de Ciências para alunos do sexto ano do ensino fundamental de uma escola pública, localizada na região Sul do Brasil. Neste laboratório foi desenvolvido um espaço para os usuários customizarem seus personagens virtuais (avatares) e foi estabelecido pelos autores do trabalho que a disposição do laboratório seria subdividida em cinco tipos diferentes de salas, separadas por tipos de materiais:

- Sala de Vídeos: nesta sala são dispostos vídeos a partir de links do YouTube em texturas, podendo ser vistos diretamente no ambiente.

- Sala de Slides e Textos: consiste em apresentações de slides e textos convertidos em imagens, que são adicionadas em texturas, que podem ser controladas com comandos de avançar e retornar.

- Sala de Questões: cada painel na sala tem questões de múltipla escolha que foram adicionadas com o uso de scripts para serem respondidas pelo usuário, em que ele escolhe uma opção e recebe um feedback.

- Sala de Simulações: o uso de softwares de modelagem 3D, como SketchUp, e, scripts de programação permitiram a modelagem de simulações animadas referentes aos tópicos abordados nesta sala.

Com relação aos conteúdos abordados, os seguintes tópicos abordados na disciplina foram selecionados: Constituição da matéria (átomos e moléculas); Fenômenos físicos e químicos; Fontes de Energia; e, Solo e Saúde. Simulações sobre estes tópicos foram 
VI Congresso Brasileiro de Informática na Educação (CBIE 2017)

Anais do XXIII Workshop de Informática na Escola (WIE 2017)

criadas, conforme pode ser observado na Figura 1, em que estão presentes experimentos tridimensionais desenvolvidos sobre os dois primeiros tópicos descritos anteriormente.

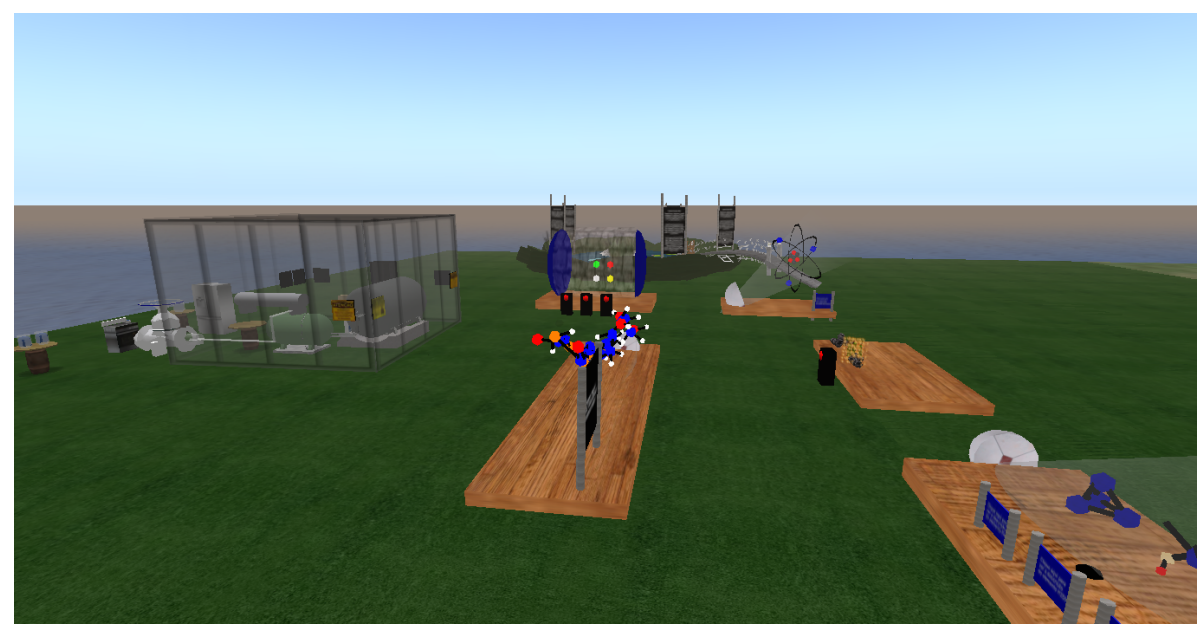

\section{Figura 1. Simulações sobre Átomos e moléculas \& Fenômenos físicos e quími- $\cos$}

Nesta ilustração é possível visualizar diferentes tipos de cadeias de átomos representadas por objetos 3D em cada painel, sendo que algumas destas são estáticas, enquanto as demais apresentam algum tipo de animação quando o usuário solicitar o início da apresentação. Também podem ser vistas simulações referentes aos estados físicos da água nos objetos à esquerda da ilustração, sendo todos estes animados.

Com relação aos participantes deste estudo de caso, foram selecionados 26 professores em formação do curso de Licenciatura em Química de uma Instituição de Ensino Superior, cujo período de experimentos com o mundo virtual ocorreu durante três semanas com encontros presenciais de aproximadamente 2 horas cada. A faixa etária predominante deste grupo está centrada até os 25 anos de idade (80\%), enquanto os demais $20 \%$ possuem 29 anos ou mais.

Com exceção de três participantes, os demais não possuem mais de um ano de experiência docente na área e nenhum dos professores havia utilizado mundos virtuais anteriormente. Como instrumento de coleta de dados, foram realizadas 3 perguntas objetivas e 3 perguntas dissertativas, adicionalmente um processo de observação direta foi adotado durante a interação com o ambiente para verificar possíveis problemas que viessem a ocorrer.

\section{Análise e Discussão dos Resultados}

O objetivo deste artigo é discorrer sobre alguns aspectos que envolvem a experiência do professor no planejamento e na construção de mundos virtuais. Para tanto, um estudo de caso foi realizado, almejando apontar os principais desafios, dificuldades e vantagens descritas pelos participantes e observadas pela prática docente usando este tipo de ambiente. Os resultados são ancorados pelo referencial teórico apresentado neste artigo, visando apresentar uma reflexão entre os testes realizados com o cenário geral descrito.

O professor selecionado para auxiliar no desenvolvimento do mundo virtual possuía conhecimentos básicos em informática, apesar disso ele não possuía conhecimentos 
VI Congresso Brasileiro de Informática na Educação (CBIE 2017)

Anais do XXIII Workshop de Informática na Escola (WIE 2017)

sobre a criação de objetos tridimensionais e programação de scripts, sendo que o mesmo não se sentiu seguro em desenvolver objetos tridimensionais no ambiente. Tal cenário pode ser descrito como costumeiro, visto que a maioria dos professores, mesmo tendo realizado cursos de capacitação em informática na educação, não terão adquirido habilidades específicas para trabalhar com este tipo de abordagem.

Tal constatação corrobora com o ponto ressaltado na terceira seção deste artigo, o qual consiste em efetuar a integração de uma equipe capacitada na área tecnológica para trabalhar conjuntamente com o docente. No contexto deste experimento, o professor selecionou os conteúdos a serem abordados e efetuou a criação dos materiais educacionais para serem inseridos pelos autores deste trabalho no mundo virtual.

No que concerne às simulações, o docente se reuniu diversas vezes para explicitar ideias e sugestões de experimentos que poderiam ser criados no ambiente, acessando o mundo virtual para verificar se estavam operando corretamente e indicar correções a serem feitas. Tal processo foi essencial para que o ambiente criado e seus conteúdos tivessem uma menor chance de terem falhas, assim como fornecer um maior grau de realidade às simulações criadas. Por se tratar de sua primeira experiência, o docente teve algumas dificuldades iniciais para aprender a utilizar o ambiente e entender seu funcionamento, assim como precisou criar uma maior diversidade de materiais educacionais do que estava comumente acostumado. Tais fatores foram superados sem maiores dificuldades, se tornando um processo mais natural no decorrer do processo de criação do ambiente.

Efetuada esta etapa do experimento, o laboratório foi utilizado pelos 26 participantes com a intenção de observar as dificuldades e os benefícios constatados por eles no uso de um mundo virtual. A curva de aprendizagem dos usuários pôde ser considerada rápida para aprender a utilizar o ambiente, visto que os mesmos interagiram sem maiores dificuldades no primeiro encontro, em que aprender a se movimentar e utilizar os comandos foram os principais obstáculos iniciais. Poucos professores buscaram experimentar a criação de alguns objetos no ambiente, enquanto que os demais centralizaram suas ações em usufruir dos recursos presentes nas salas e entender seu funcionamento. Com relação aos problemas que foram identificados durante o experimento e apontados pelos usuários na questão dissertativa, a qualidade da conexão com a Internet foi o principal fator identificado.

A maioria dos usuários apontou dificuldades em movimentar o personagem e principalmente assistir aos vídeos disponibilizados no ambiente, visto que a conexão não apresentava boa qualidade e este tipo de atividade exigia uma largura de banda maior para operar corretamente, o que afetou diretamente na interação dos usuários no ambiente. Este fator está diretamente relacionado ao ponto descrito por [Fernandes et al. 2007], em que a baixa velocidade da Internet acaba por prejudicar a interação no ambiente e limitar o uso de seus recursos.

Tal problema acaba por ser recorrente, conforme experiências anteriores vividas pelos autores deste artigo, e, também apontado nas pesquisas de [Antonello et al. 2009] e [Oliver et al. 2010]. Tanto o professor quanto os alunos podem se sentir desmotivados em usar o ambiente, sendo este fator essencial no planejamento da infraestrutura tecnológica necessária.

Apesar das dificuldades observadas, 19 participantes concordaram totalmente e 5 
VI Congresso Brasileiro de Informática na Educação (CBIE 2017)

Anais do XXIII Workshop de Informática na Escola (WIE 2017)

parcialmente que o uso dos recursos didáticos e simulações no mundo virtual possa vir a auxiliar no processo de ensino da disciplina. Em consonância com este questionamento, 20 participantes também disseram que poderiam utilizar os mundos virtuais em outros momentos em práticas pedagógicas.

A manipulação dos objetos 3D, recursos multimídias foi encarada pelos participantes de forma adequada, em que os mesmos ressaltaram que conseguiram de forma intuitiva entender o funcionamento destes recursos no ambiente. Isso se torna importante, visto que eles consideraram essencial a diversidade de materiais possíveis de serem usados no mundo virtual, o que acarreta na expansão de possibilidades a serem exploradas em atividades com os alunos.

Os dados mostram que os professores entenderam que as dificuldades identificadas podem ser superadas e assimiladas, apostando no potencial dos recursos ofertados por esse tipo de ambiente. Este ponto é instigante, visto que conforme apontado por [Jacka 2015], os mundos virtuais ainda estão em processo de construção e os docentes precisarão passar por este processo inicial mais oneroso, para posteriormente utilizar os recursos e benefícios que o ambiente pode proporcionar.

Este ponto é reforçado nos dados obtidos junto aos participantes, em que 25 deles assinalaram terem ficado motivados em utilizar futuramente os mundos virtuais em sua prática docente. Se torna importante lembrar que para este processo, será necessário efetuar o planejamento das atividades educacionais seguindo objetivos claros e uma metodologia bem estabelecida, ancorada por conceitos teóricos da educação. Trabalhos como o de [Bouda 2012], [Soto 2013] e [Nelson and Erlandson 2012] abordam o processo de design e planejamento de atividades didáticas nos mundos virtuais, sendo um processo essencial na prática docente a definição adequada das tarefas.

Exemplo disto está no comentário feito por um dos participantes: "Acho que me ajudou a ter ideias de aulas diferenciadas para os alunos e também de mostrar uma nova forma de ajudá-los a compreender assuntos, muitas vezes complexos". Características como a imersividade, colaboratividade, autoria, interação mais ativa e retorno imediato das ações desempenhadas fazem com que o mundo virtual estabeleça um leque variado de alternativas a serem articuladas pelo professor.

Desta forma, os comentários apresentados nas questões dissertativas sobre os benefícios e a visão geral deste tipo de prática na docência foram predominantemente positivos. Os principais pontos assinalados foram a interação experimental que o mundo virtual oferece ao usuário, envolvendo o uso da tecnologia na educação, o que pode tornar o ensino mais interessante e motivador para os alunos, servindo como um incentivo para eles aplicar o conhecimento de forma mais prática.

Também foi descrito que este tipo de projeto pode ser visto como interessante e instigador para o professor, dada a diversidade de alternativas que podem ser adotadas na prática pedagógica com o uso deste ambiente e o quanto pode ajudar na prática de tópicos mais complexos de serem ensinados e que exigem uma representação visual mais realista.

\section{Considerações Finais}

A crescente utilização de novos recursos tecnológicos no meio educacional tem proporcionado a experimentação de novas técnicas pedagógicas e o uso de ambientes virtuais de 
VI Congresso Brasileiro de Informática na Educação (CBIE 2017)

Anais do XXIII Workshop de Informática na Escola (WIE 2017)

aprendizagem para apoiar o processo de aprendizagem dos estudantes. Nesse contexto, os mundos virtuais podem ser vistos como uma forma de complementar os conteúdos vistos em sala de aula ou em disciplinas ministradas a distância, em diferentes áreas de ensino.

O presente artigo buscou apresentar uma visão esclarecedora dos principais benefícios e desafios que estão presentes na prática docente, no que concerne ao uso dos mundos virtuais. Para tanto, foi efetuada uma análise crítica desde o referencial teórico descrito neste trabalho, juntamente com um estudo de caso voltado para averiguar aspectos relacionados à experiência do docente.

A migração do ensino tradicional para o uso de novas tecnologias, como os mundos virtuais, é ainda um desafio para muitos docentes, em que para encontrar a solução será necessário trabalhar de forma conjunta com pessoas capacitadas na área de tecnologia, mesclando conhecimentos tecnológico com o planejamento didático adequado ao tipo de atividade que será realizado. O estudo de caso buscou apresentar alguns dos pontos ressaltados neste artigo, mostrando que há um conhecimento maior das principais dificuldades enfrentadas na prática docente neste tipo de ambiente.

[Christensen et al. 2013] ressaltam que nos próximos 5 a 10 anos, a expansão dos mundos virtuais na educação tem um potencial instigador para mudar diversas formas de aprendizagem em diferentes áreas educacionais. O caminho que tem sido percorrido está em consonância com o que foi descrito pelos autores, em que os docentes em sua prática pedagógica terão que superar as atuais barreiras no uso deste tipo de ambiente, para que possam usufruir mais naturalmente e com menores dificuldades das vantagens que os mundos virtuais oferecem. Como pesquisas futuras, está o desenvolvimento de interfaces adaptativas e mecanismos de interconexão entre o mundo virtual e o ambiente de aprendizagem Moodle, para que a inserção de conteúdos, como slides, vídeos e questões possam ser feitas de forma mais automatizada pelo docente, reduzindo a carga de trabalho dele e da equipe tecnológica.

\section{Referências}

Antonello, R., Fernandes, S., Moreira, J., Cunha, P., Kamienski, C., and Sadok, D. (2009). Traffic analysis and synthetic models of second life. Multimedia Systems, 15(1):33-47.

Avila, B. G., Tarouco, L. M. R., Passerino, L. M., and Guterer, P. (2014). Autoria nos mundos virtuais: um novo desafio ao docente. Revista Novas Tecnologias na Educação, 12(2):01-10.

Bouda, T. (2012). User-centered design and evaluation of virtual worlds. In Experiential Learning in Virtual Worlds: 2nd Global Conference, pages 01-11. Inter-Disciplinary. Net.

Christensen, I.-M. F., Marunchak, A., and Stefanelli, C. (2013). Added Value of Teaching in a Virtual World, pages 125-137. Palgrave Macmillan UK, London.

Fernandes, S., Antonello, R., Moreira, J., Sadok, D., and Kamienski, C. (2007). Traffic analysis beyond this world: the case of second life. In 17th International workshop on Network and operating systems support for digital audio and video, University of Illinois, Urbana-Champaign, pages 4-5.

Fullan, M. (2013). Commentary: The new pedagogy: Students and teachers as learning partners. Learning Landscapes, 6(2):23-29. 
VI Congresso Brasileiro de Informática na Educação (CBIE 2017)

Anais do XXIII Workshop de Informática na Escola (WIE 2017)

Gregory, S., Scutter, S., Jacka, L., McDonald, M., Farley, H., and Newman, C. (2015). Barriers and enablers to the use of virtual worlds in higher education: An exploration of educator perceptions, attitudes and experiences. Journal of Educational Technology \& Society, 18(1):03-12.

Griol, D., Molina, J. M., and Callejas, Z. (2014). An approach to develop intelligent learning environments by means of immersive virtual worlds. J. Ambient Intell. Smart Environ., 6(2):237-255.

Jacka, L. (2015). Virtual worlds in pre-service teacher education : the introduction of virtual worlds in pre-service teacher education to foster innovative teaching-learning processes. $\mathrm{PhD}$ thesis, Southern Cross University.

Johnson, C., Vorderstrasse, A., and Shaw, R. (2009). Virtual worlds in health care higher education. Journal For Virtual Worlds Research, 2(2):01-12.

Kozlovsky, E. and Kravtsov, H. (2011). Virtual laboratory for distance learning: Conceptual design and technology choices. In Proceddings of the ICT in Education, Research and Industrial Applications: Integration, Harmonization and Knowledge Transfer (ICTERI), pages 116-125.

Nelson, B. C. and Erlandson, B. E. (2012). Design for learning in virtual worlds. Taylor and Francis.

Nunes, F. B., Herpich, F., Paschoal, L. N., Lima, J. V. D., and Tarouco, L. M. R. (2016a). Systematic review of virtual worlds applied in education. In Proceedings of the XXVII Brazilian Symposium on Computers in Education (SBIE 2016), pages 657-666.

Nunes, F. B., Herpich, F., Zunguze, M. C., and de Lima, J. V. (2016b). Utilização do mastery learning em sala de aula: uma abordagem integrada aos mundos virtuais. In Anais do $24^{\circ}$ Workshop sobre Educação em Computação (CSBC 2016), pages 24422451.

Oliver, I. A., Miller, A. H., and Allison, C. (2010). Virtual worlds, real traffic: Interaction and adaptation. In Proceedings of the First Annual ACM SIGMM Conference on Multimedia Systems, MMSys '10, pages 305-316, New York, NY, USA. ACM.

Prensky, M. (2001). Digital natives, digital immigrants. On the Horizon, 9(5):1-6.

Soto, V. J. (2013). Which instructional design models are educators using to design virtual world instruction? Journal of Online Learning and Teaching, 9(3):364.

Twining, P., Raffaghelli, J., Albion, P., and Knezek, D. (2013). Moving education into the digital age: the contribution of teachers' professional development. Journal of Computer Assisted Learning, 29(5):426-437.

Zanotto, D., Rolim, C., and Fernandes, S. (2009). Desenvolvimento de um surveybot em mundos virtuais com aplicação em sistemas de avaliação. In Anais do XVII Workshop sobre Educação em Informática, pages 623-626. 